\section{SP 05 IMPLEMENTING A TRAINING AND ACCREDITATION SCHEME FOR TTA (TO TAKE AWAY) PRE-PACK DISPENSING}

Rania Betmouni, Nusret Gillani. BUPA Cromwell Hospital

\subsection{6/archdischild-2015-308634.5}

Background The Paediatric Walk In Centre (PWIC) opened in March 2014 and is open Monday to Friday (5-9 pm), Saturday (10 am-8 pm) and Sunday (10 am-6 pm). To facilitate dispensing out of hours, TTA pre-packs were stocked in the centre. As the paediatric staff do not have the same skills as pharmacy staff in screening and dispensing an accreditation was developed to train staff working in the PWIC.

Aim To implement a safe dispensing process for paediatric staff working in the PWIC.

Method To ensure the dispensing process occurred safely, a limited list of TTA pre-packs with approved labels was agreed with the lead clinician. The pharmacy developed a Nurse Led Dispensing Policy that included dispensing and checking standards (double check by two staff members not involved in the prescribing of the medicine), dispensing and checking record log, clinical screening standards, drug monograph for clinical screen and managing medication incidents. The accreditation programme consisted of two parts:

Training consisting of an hour long presentation on safe prescribing, dispensing and checking. The presentation highlighted the principles of prescribing in paediatrics including: calculating doses; the importance of clinical screening (reviewing the layout of drug monographs in BNFC); legal requirements for dispensing/providing information (valid prescriptions and dispensing labels) and finally medication safety and how errors can be avoided. All staff were expected to attend the presentation.

Accreditation consisted of supervised open book exercises. Staff were expected to accurately clinically screen five prescriptions, dispense ten items and check four dispensed items. They were then asked to deal with a dispensing error scenario.

All staff had to achieve $100 \%$ in each of the exercises. If staff failed they were asked to repeat the accreditation with new exercises.

When the Centre opened the use of TTA pre-packs was monitored in the first few weeks to help identify any further training needs.

Result 21 staff members have completed and passed the accreditation. Five staff members had to repeat the exercises. Reasons for failing the accreditation included missing a penicillin allergy, wrong name on the dispensing label, omitting a patient information leaflet, wrong expiry of reconstituted antibiotic and wrong calculation of dose to administer resulting in wrong directions on the label. In the initial period, the TTA pre-packs of paracetamol and ibuprofen were inadvertently used for stat administration of analgesia in the Centre. Staff were reminded regular unlabelled stock should be used for medicines administration and not TTA prepacks. Also, due to staff shortage, a prescriber was involved in the final check. No medication safety incidents have been reported.

Conclusion The training and accreditation programme has allowed for the implementation of a safe dispensing and checking process for TTA prepacks by medical and nursing staff. The next step is to audit the process. 